NBER WORKING PAPER SERIES

\title{
NONPARAMETRIC IDENTIFICATION AND ESTIMATION OF NONADDITIVE HEDONIC MODELS
}

\author{
James J. Heckman \\ Rosa L. Matzkin \\ Lars Nesheim \\ Working Paper 15226 \\ http://www.nber.org/papers/w15226
}

\author{
NATIONAL BUREAU OF ECONOMIC RESEARCH \\ 1050 Massachusetts Avenue \\ Cambridge, MA 02138 \\ August 2009
}

This research was supported by NSF grants SES-0241858 and BCS-0433990. Lars Nesheim also thanks the Leverhulme Trust and the UK Economic and Social Research Council (ESRC grant RES-589-28-0001) for support through its funding of the Centre for Microdata Methods and Practice(http://www.cemmap.ac.uk). Rosa L. Matzkin research has been supported also by NSF grant SES-0551272. We thank the participants of seminars at Northwestern University, Universidad de San Andres, Princeton University, Harvard/MIT, UCLA/USC, University of California at Berkeley, University of Minnesota, the Bureau of Labor Statistics, University of Chicago, Universidad Di Tella, the 2002 Workshop on Characteristics Models: Theory and Applications (University of Copenhagen), the 2002 Workshop on Mathematical Economics (IMPA), and the 2004 Banff International Research Station (BIRS) workshop on "Mathematical Structures in Economic Theory and Econometrics" for their useful comments. We thank Myrna Wooders and Daniel McFadden for many stimulating conversations, and Donald J. Brown for specific comments. The views expressed herein are those of the author(s) and do not necessarily reflect the views of the National Bureau of Economic Research.

NBER working papers are circulated for discussion and comment purposes. They have not been peerreviewed or been subject to the review by the NBER Board of Directors that accompanies official NBER publications.

(C) 2009 by James J. Heckman, Rosa L. Matzkin, and Lars Nesheim. All rights reserved. Short sections of text, not to exceed two paragraphs, may be quoted without explicit permission provided that full credit, including $\odot$ notice, is given to the source. 
Nonparametric Identification and Estimation of Nonadditive Hedonic Models

James J. Heckman, Rosa L. Matzkin, and Lars Nesheim

NBER Working Paper No. 15226

August 2009

JEL No. C14,D41,D58

\section{ABSTRACT}

This paper studies the identification and estimation of preferences and technologies in equilibrium hedonic models. In it, we identify nonparametric structural relationships with nonadditive heterogeneity. We determine what features of hedonic models can be identified from equilibrium observations in a single market under weak assumptions about the available information. We then consider use of additional information about structural functions and heterogeneity distributions. Separability conditions facilitate identification of consumer marginal utility and firm marginal product functions. We also consider how identification is facilitated using multimarket data.

James J. Heckman

Department of Economics

The University of Chicago

1126 E. 59th Street

Chicago, IL 60637

and NBER

jjh@uchicago.edu

Rosa L. Matzkin

Department of Economics

University of California, Los Angeles

8283 Bunche Hall

Mail Stop 147703

Los Angeles CA 90095

matzkin@econ.ucla.edu
Lars Nesheim

Department of Economics

University College London

Gower Street

London WC1E 6BT

United Kingdom

1.nesheim@ucl.ac.uk 


\section{Introduction}

How much are consumers willing to pay for increased product quality? What wage premium do workers require to compensate them for an increase in on-the-job fatality risk? How much are homeowners willing to pay to avoid moving one mile further from work? What will be the effect of new clean air legislation on real estate prices? Hedonic models provide information that helps to narrow the range of possible answers to these questions. In an hedonic market, the price function for a good or service decomposed into its attributes measures the equilibrium relationship between attributes of the good and the price at which buyers and sellers are willing to trade. It is the price that equates supply and demand in the space of hedonic attributes. It provides an exact measure of the marginal willingness to pay and willingness to accept for equilibrium transactions in a market. However, in general, the equilibrium price function does not identify the willingness to pay for counterfactual transactions that might occur in markets characterized by different endowments, technology and preferences. Due to unobserved heterogeneity, there is a selection problem. ${ }^{1}$ To take a labor market example, workers who choose to work in jobs with a low fatality risk are likely to have low unobserved tolerance for risk. As a result, the compensation they require to accept more risk is likely to be higher than the equilibrium wage premium for risky jobs. To identify willingness to pay and willingness to accept for counterfactual transactions, one needs to identify the structural parameters of buyers and sellers.

Knowledge of the hedonic price function alone is not enough to analyze general equilibrium effects of policy changes in hedonic markets. When supply or demand conditions change, buyers and sellers will in general alter how they sort in hedonic markets. Hence, to analyze general equilibrium changes in hedonic markets, it is necessary to identify the structural parameters. $^{2}$

Observed and unobserved heterogeneity play key roles in hedonic equilibrium sorting models. In particular their distributions and the underlying utility and production functions of the agents shape equilibrium sorting outcomes and shape how a hedonic economy will react to changes in the economic environment. Previous studies of hedonic markets have analyzed either parametric or restrictive nonparametric utility and production functions, where the unobserved heterogeneity is specified as a term added to marginal utility or marginal product functions. ${ }^{3}$ Ekeland, Heckman and Nesheim (2004) analyze nonparametric hedonic

\footnotetext{
${ }^{1}$ See, e.g., Rosen (1974).

${ }^{2}$ See, e.g., Hurwicz (1962) or, for a more modern statement, Heckman and Vytlacil (2007).

${ }^{3}$ For analysis of identification of parametric hedonic models, see Brown and Rosen (1982), Brown (1983),
} 
models with additive marginal utility and additive marginal product functions. They show that hedonic models with additivity restrictions are nonparametrically identified with single market data and present two methods for recovering the structural functions in such models.

The additivity assumptions used to establish identification in Ekeland, Heckman, and Nesheim (2004) are strong. No heterogeneity in the curvature of production and preference functions is allowed. In addition, these restrictions are over-identifying.

In contrast, this paper establishes nonparametric identification of structural functions and distributions in general hedonic models without imposing additivity. We allow the curvature of the marginal utility for the product attribute, as well as the distribution of the marginal utilities, to vary in general ways across agents with different observed characteristics. We allow an analogous property to hold for nonadditive unobserved heterogeneity in marginal product functions on the supply side of the market.

We first analyze what can be identified in an unrestricted model fit using data from a single market that satisfies standard economic regularity conditions and additional mild statistical regularity conditions. We then restrict the functional structure of the marginal utilities and marginal product functions, obtaining nonparametric generalizations of random coefficient and household scale models. We show that in these cases, the marginal utility and marginal product functions, as well as the distribution of the unobserved characteristics of the agents, are identified. Our proofs of identification are constructive and suggest nonparametric estimation strategies whose properties we examine in a web appendix. ${ }^{4}$

We go on to show that models that cannot be identified in a single cross section are identified using data from multiple markets. This follows from the economics of the hedonic model. In general, variation in the distributions of observed variables across markets induces cross-market variation in price functions. This part of our analysis formalizes and extends discussions in Rosen (1974), Brown and Rosen (1982), Epple (1987) and Kahn and Lang (1988).

To focus on the key identification problems that arise in a setup where marginal utilities are nonadditive in unobserved heterogeneity, we concentrate on an hedonic model with a single product characteristic. Our main results can be extended to hedonic models with multiple characteristics captured by an index structure, as in Epple and Sieg (1999) and Sieg, Smith, Banzhaf, and Walsh (2004). Our methodology extends the two stage method of Rosen (1974) to a nonparametric setting. The first stage estimates the hedonic function and Epple (1987), Bajari and Benkard (2005), or Berry and Pakes (2007).

${ }^{4}$ See Web Appendices D and F at http://jenni.uchicago.edu/nonpara_hedonic 
its derivatives. Using the estimated price function together with the first order conditions of the agents identifies production and utility functions.

This paper proceeds in the following way. Section 2 describes the hedonic model for a product with a single attribute. Section 3 studies the identification of nonadditive marginal utility and nonadditive marginal product functions. Section 4 analyzes identification in multiple markets. Analog estimators are discussed in Section 5. Their properties are derived in the web appendix (see http://jenni.uchicago.edu/nonpara_hedonic) for this paper. The web appendix also presents a limited Monte Carlo analysis. Section 6 summarizes and discusses possible extensions.

\section{The Competitive Hedonic Equilibrium Model}

The model analyzed in this paper applies to a spot market in which products are differentiated by their attributes, prices are set competitively and participating buyers and sellers each trade a single type of product chosen from a set of feasible products. Our analysis of demand in this section, and in Section 3, applies equally to hedonic demand estimation problems in imperfectly competitive markets where consumers are fully informed price takers. The multi-market analysis in Section 4 can readily be adapted to study demand in imperfectly competitive markets as long as there is sufficient cross-market variation in market structure to induce price variation. We focus on competitive markets for ease of exposition.

To fix ideas, we consider a labor market setting in which jobs are characterized by their attributes. Workers (sellers) match to single employee firms (buyers) and supply job services indexed by a scalar $z$ where $z \in \widetilde{Z}{ }^{5}$ We assume that $\widetilde{Z} \subseteq \mathbf{R}$ is compact. The space $\widetilde{Z}$ is the space of technologically feasible job attributes. The variable $z$ denotes a job attribute assumed to be a disamenity for the workers and an input for the firms. For example, z could measure the probability of injury on the job as in Kniesner and Leeth (1995) or Leeth and Ruser (2003), so $\widetilde{Z}=[0,1]$. Let $P(z)$ be a twice continuously differentiable price function defined on $\widetilde{Z}$. The value of $P(z)$ is the wage paid at a job characterized by attribute $z$.

Each worker has quasilinear utility function $P(z)-U(z, x, \varepsilon)$ where $x$ is a vector of

\footnotetext{
${ }^{5}$ If jobs are characterized by a vector of attributes $a \in \widetilde{A} \subseteq \mathbf{R}_{n}$, we assume that preferences and costs depend on $a$ only through the scalar index $z=g(a)$. We discuss identification of a single index model in Section 3. Alternatively, if utility is quasilinear and both utility and production functions are additively separable functions of $z$ and other attributes, the price function will be as well and the market for $z$ can be analysed independently of the other attributes.
} 
observed characteristics of dimension $n_{x}$ and $\varepsilon$ is a scalar unobserved heterogeneity term. ${ }^{6}$ Observe that $U(\cdot)$ is a disutility. $\varepsilon$ is statistically independent of $x$. In the risk of injury example, $\varepsilon$ is interpreted as the component of unobserved preference for risk that is independent of observables such as age, education, gender, race, etc. Note that since $\varepsilon$ is allowed to enter marginal utility $U_{z}$ in a nonadditive way, the independence assumption is much weaker than it would be if $\varepsilon$ entered additively. In particular, the nonadditive specification is consistent with a model where $U_{z}$ is a function of an unobserved $\xi=m(x, \varepsilon)$, whose distribution depends on the vector of observed characteristics, $x$, of the agent. The population of workers is described by the pair of density functions $f_{x}$ and $f_{\varepsilon}$, which are assumed to be strictly positive on the compact sets $\widetilde{X} \subseteq \mathbf{R}^{n_{x}}$ and $\widetilde{E} \subseteq \mathbf{R}$, respectively. Each worker may choose not to trade, in which case they obtain reservation utility $V_{0}$.

Each firm has a production function $\Gamma(z, y, \eta)$ where $y$ is a vector of observed characteristics of the firm of dimension $n_{y}$ and $\eta$ is a scalar unobserved heterogeneity term. We assume that $\eta$ is statistically independent of $y$, and that $(y, \eta)$ is independent of $(x, \varepsilon)$. The population of firms is described by the pair of density functions $f_{y}$ and $f_{\eta}$ that are strictly positive on the compact sets $\widetilde{Y} \subseteq \mathbf{R}^{n_{y}}$ and $\widetilde{H} \subseteq \mathbf{R}$, respectively. If a firm opts out of the market, it earns reservation profits $\Pi_{0}$.

Both $U$ and $\Gamma$ are assumed to be twice continuously differentiable with respect to all arguments. ${ }^{7}$ Additionally, we assume that, given the equilibrium price function, each worker and firm that participates has a unique interior optimum. A sufficient condition for this to hold is a Spence-Mirrlees type single-crossing condition, requiring that $U_{z z}-\Gamma_{z z}>0$, $U_{z \varepsilon}<0$, and $\Gamma_{z \eta}>$, for all $(x, \varepsilon, y, \eta)$, be satisfied. ${ }^{8}$ For simplicity, we assume that these conditions are satisfied.

Each worker chooses $z \in \widetilde{Z}$, a job type or a location in the space of job attributes, to maximize

$$
P(z)-U(z, x, \varepsilon)
$$

From uniqueness of the optimum, it follows from Gale and Nikaido (1965) that there exists

\footnotetext{
${ }^{6}$ When utility is not quasilinear, we can identify the ratio of the marginal utility of $z$ to the marginal utility of income (See Web Appendix B.).

${ }^{7}$ The equilibrium analysis can be extended to cases in which $z$ is a vector, the arguments of the functions are discrete, the measures on characteristics are not absolutely continuous with respect to Lebesgue, or the functions are not differentiable. See Ekeland (2005) or Chiappori, McCann, and Nesheim (2008) for details.

${ }^{8}$ See Chiappori, McCann, and Nesheim (2008) for details.
} 
a supply function $z=s(x, \varepsilon)$ such that

$$
P_{z}(s(x, \varepsilon))-U_{z}(s(x, \varepsilon), x, \varepsilon)=0
$$

By the Implicit Function Theorem,

$$
\frac{\partial s(x, \varepsilon)}{\partial \varepsilon}=\frac{U_{z \varepsilon}(s(x, \varepsilon), x, \varepsilon)}{P_{z z}(s(x, \varepsilon))-U_{z z}(s(x, \varepsilon), x, \varepsilon)}
$$

so that $\frac{\partial s(x, \varepsilon)}{\partial \varepsilon}>0$ since $U_{z \varepsilon}<0$ and the denominator is negative from the assumption of an interior optimum. Our assumptions imply that $s(x, \varepsilon)$ is strictly monotonic in $\varepsilon$ and that its inverse with respect to $\varepsilon$ exists. We denote the inverse supply function by $\varepsilon=\widetilde{s}(z, x)$.

A parallel analysis can be performed for the demand side of the market. Each firm chooses $z \in \widetilde{Z}$ to maximize the profit function

$$
\Gamma(z, y, \eta)-P(z)
$$

The resulting demand function $z=d(y, \eta)$ satisfies

$$
\Gamma_{z}(d(y, \eta), y, \eta)-P_{z}(d(y, \eta))=0
$$

and

$$
\frac{\partial d(y, \eta)}{\partial \eta}=\frac{\Gamma_{z \eta}(d(y, \eta), y, \eta)}{P_{z z}(d(y, \eta))-\Gamma_{z z}(d(y, \eta), y, \eta)}
$$

so that $\frac{\partial d(y, \eta)}{\partial \eta}>0$ since $\Gamma_{z \eta}>0$ and the denominator is positive. We denote the inverse demand function $\eta=\widetilde{d}(z, y)$.

The inverse supply and demand functions characterize equilibrium. ${ }^{9}$ Let

$$
Z_{s}=\{z \in \widetilde{Z} \mid z=s(x, \varepsilon) \text { for some }(x, \varepsilon) \in \widetilde{X} \times \widetilde{E}\}
$$

be the range of the mapping $s(x, \varepsilon)$. Using a standard change of variables formula, the density of supply implied by the inverse supply function is

$$
\int_{\widetilde{X}} f_{\varepsilon}(\widetilde{s}(z, x)) f_{x}(x) \frac{\partial \widetilde{s}(z, x)}{\partial z} d x
$$

\footnotetext{
${ }^{9}$ See Ekeland, Heckman, and Nesheim (2004).
} 
for $z \in Z_{s}$. For $z \in \widetilde{Z} \backslash Z_{s}$, the density of supply is zero.

Analogously, let

$$
Z_{d}=\{z \in \widetilde{Z} \mid z=d(y, \eta) \text { for some }(y, \eta) \in \widetilde{Y} \times \widetilde{H}\}
$$

be the range of the mapping $d(y, \eta)$. The density of the demanded $z$ is

$$
\int_{\widetilde{Y}} f_{\eta}(\widetilde{d}(z, y)) f_{y}(y) \frac{\partial \widetilde{d}(z, y)}{\partial z} d y
$$

for $z \in Z_{d}$. For $z \in \widetilde{Z} \backslash Z_{d}$, the density of demand is zero. Both inverse supply and inverse demand depend on $P(z)$ although the dependence is implicit.

Among the set of smooth price functions that yield unique interior optima, an equilibrium price function must satisfy the condition that the density of supply equals the density of demand for all values of $z \in \widetilde{Z}$. Making explicit the dependence of the supply and demand densities on $P$, this condition requires that $Z_{s}=Z_{d}$ and that

$$
\int_{\widetilde{X}} f_{\varepsilon}(\widetilde{s}(z, x ; P(z))) f_{x}(x) \frac{\partial \widetilde{s}(z, x ; P(z))}{\partial z} d x=\int_{\widetilde{Y}} f_{\eta}(\widetilde{d}(z, y ; P(z))) f_{y}(y) \frac{\partial \widetilde{d}(z, y ; P(z))}{\partial z} d y
$$

for all $z \in Z_{s} \cap Z_{d}$, where $P(z)$ is the price function. The equilibrium price function solves this equation.

\subsection{Properties of Equilibrium}

Ekeland (2005) and Chiappori, McCann, and Nesheim (2008) show that an equilibrium exists in the hedonic model under very general conditions which include those given in the previous section. They also show that the single-crossing property presented in the previous section is sufficient for equilibrium to be unique and pure in the sense that each worker matches to a single firm and each pair chooses a single job type $z$. This implies, in particular, that counterfactual policy analysis using this model provides unambiguous answers.

A key implication of the model is that cross-market variation in the price function is determined by cross-market variation in the functions $\left(f_{x}, f_{\varepsilon}\right)$ and $\left(f_{y}, f_{\eta}\right)$. To see this more 
clearly, substitute for $\frac{\partial \widetilde{s}(z, x)}{\partial z}$ and $\frac{\partial \widetilde{d}(z, y)}{\partial z}$ and rewrite $(2.5)$ as

$$
P_{z z}(z)=\frac{\int_{\widetilde{Y}} f_{\eta} f_{y} \frac{\Gamma_{z z}}{\Gamma_{z \eta}} d y-\int_{\widetilde{X}} f_{\varepsilon} f_{x} \frac{U_{z z}}{U_{z \varepsilon}} d x}{\int_{\widetilde{Y}} \frac{f_{\eta} f_{y}}{\Gamma_{z \eta}} d y-\int_{\widetilde{X}} \frac{f_{\varepsilon} f_{x}}{U_{z \varepsilon}} d x}
$$

where the arguments of the functions are suppressed. This second order differential equation depends on the objects $(U, \Gamma),\left(f_{x}, f_{\varepsilon}\right)$ and $\left(f_{y}, f_{\eta}\right)$. If the parameters $(U, \Gamma)$ and $\left(f_{\varepsilon}, f_{\eta}\right)$ are fixed while $\left(f_{x}, f_{y}\right)$ vary across markets, the equilibrium price will vary across markets. We exploit the variation induced by these changes in Section 4 to show identification of the structural parameters using multimarket data.

Finally, our assumptions imply that the joint distribution of observables $(z, x, y)$ implied by the model will be continuous. Since the distributions of $(x, \varepsilon)$ and $(y, \eta)$ are continuous, the joint distribution of $(z, x, y)$ will be continuous if almost all agents do not bunch at a single point in $\widetilde{Z}$. Given a point $z_{b} \in \widetilde{Z}$, it is possible that a positive measure of agents will bunch at $z_{b}$, only if the set

$$
B=\left\{(x, \varepsilon),(y, \eta) \mid \Gamma_{z}\left(z_{b}, y, \eta\right)=U_{z}\left(z_{b}, x, \varepsilon\right)\right\}
$$

has positive measure. When $(x, \varepsilon)$ and $(y, \eta)$ are continuously distributed, this set cannot have positive measure if $\Gamma_{z \eta}>0$ and $U_{z \varepsilon}<0$. Our assumed single-crossing condition rules out bunching and implies that $(z, x, y)$ are continuously distributed on their respective domains. ${ }^{10}$

\section{Identification in a Single Market}

This section discusses identification of supply function $s(x, \varepsilon)$, the marginal utility function $U_{z}(z, x, \varepsilon)$ and the distribution of $\varepsilon$ in a single market. We do not discuss the demand side of the market because the analysis for that case is analytically similar. Our analysis assumes that the equilibrium price function $P(z)$ and the distribution of $(z, x)$ are known where $z$ denotes the observed hedonic location choice of an individual and $x$ denotes the vector of observed worker characteristics. Estimation of $P(z)$ does not affect the identification analysis. In Section 5, we discuss how it impacts the estimation analysis. (See Web Appendix

\footnotetext{
${ }^{10}$ Examples with bunching can be generated either by allowing for mass points in the distributions or worker or firm types or by relaxing the single-crossing condition. Further analysis of bunching is available in Web Appendix C.
} 


\section{A.)}

We conduct the analysis for the case where $z \in \mathbf{R}$. The analysis applies equally to the case where $z=g(a) \in \mathbf{R}$ and $a \in \mathbf{R}^{n}$ subject to a normalization on $g$. For example, if $z=\beta \cdot a$ and $\beta_{1}=1$, then the vector of parameters for $\beta$ can be estimated from the price function since the equilibrium price has the form $p=P(\beta \cdot a)$. Under these conditions $\frac{\frac{d P}{d a_{j}}}{\frac{d P}{d a_{1}}}=\beta_{j}$. Once $\beta$ has been identified, the analysis in this paper can be applied to $z=\beta \cdot a \cdot{ }^{11}$

In nonadditive hedonic models, supply function $s(x, \varepsilon)$ is a nonseparable function of a vector of observables $x$ and a scalar unobservable $\varepsilon$. While we assume that $\varepsilon$ is independent of $x$, nonetheless, the nonadditive structure allows for arbitrary interactions between $x$ and $\varepsilon$ in producing outcomes. Furthermore, the theoretical structure of Section 2 implies that $s$ is a strictly increasing function of $\varepsilon$. The function $s(x, \varepsilon)$ can then be identified using results from Matzkin (1999) and Matzkin (2003), where it is shown that, using the independence between $\varepsilon$ and $x$, and the strict monotonicity of $s$ in $\varepsilon$, one has that for all values $x$ of $X$ and all values $\varepsilon^{\prime}$ of $\varepsilon$,

$$
\begin{aligned}
F_{\varepsilon}\left(\varepsilon^{\prime}\right) & =\operatorname{Pr}\left(\varepsilon \leq \varepsilon^{\prime}\right) \\
& =\operatorname{Pr}\left(\varepsilon \leq \varepsilon^{\prime} \mid x\right) \\
& =\operatorname{Pr}\left(s(X, \varepsilon) \leq s\left(x, \varepsilon^{\prime}\right) \mid X=x\right) \\
& =F_{Z \mid X=x}\left(s\left(x, \varepsilon^{\prime}\right)\right) .
\end{aligned}
$$

The conditional distribution function, $F_{Z \mid X=x}$, of $Z$ given $X=x$, is strictly increasing when the density of $\varepsilon$ is everywhere positive. In this case,

$$
s\left(x, \varepsilon^{\prime}\right)=F_{Z \mid X=x}^{-1}\left(F_{\varepsilon}\left(\varepsilon^{\prime}\right)\right) .
$$

Note that $s(x, \varepsilon)$ is a reduced form. It depends on the structural utility function and on the equilibrium price $P(z)$. Nevertheless, it can be used to predict partial equilibrium impacts of changes in $x$ or $\varepsilon$ on equilibrium outcomes. Moreover, identification of $s(x, \varepsilon)$ is a first step required for identification of $U_{z}$.

Identification of $s(x, \varepsilon)$ requires either a normalization of the function (e.g., fixing its values at a particular value of $X$ ) or of the distribution of the unobserved $\varepsilon$ (e.g., assuming that the distribution $F_{\varepsilon}\left(\varepsilon^{\prime}\right)$ is known). If, for example, we normalize $s$ so that at a value $\bar{x}$

\footnotetext{
${ }^{11}$ Epple and Sieg (1999) and Sieg, Smith, Banzhaf, and Walsh (2004) assume this index structure in their analysis of parametric hedonic models.
} 
of $X$, and all $\varepsilon^{\prime}$,

$$
s\left(\bar{x}, \varepsilon^{\prime}\right)=\varepsilon^{\prime}
$$

then one gets from the above equations that for all $\varepsilon^{\prime}$

$$
F_{\varepsilon}\left(\varepsilon^{\prime}\right)=F_{Z \mid X=\bar{x}}\left(s\left(\bar{x}, \varepsilon^{\prime}\right)\right)
$$

and for all $x$

$$
s\left(x, \varepsilon^{\prime}\right)=F_{Z \mid X=x}^{-1}\left(F_{Z \mid X=\bar{x}}\left(\varepsilon^{\prime}\right)\right) .
$$

See Matzkin (1999) or Matzkin (2003) for details.

Certain features of the function $s$, such as $\partial s\left(x, \varepsilon^{\prime}\right) / \partial x$, the partial derivative of $s$ with respect to $x$, or $z_{1}-z_{0}=s\left(x_{1}, \varepsilon\right)-s\left(x_{0}, \varepsilon\right)$, the change in $z$ due to a change in $x$ from $x_{0}$ to $x_{1}$, leaving the value of $\varepsilon$ fixed, are invariant to the choice of a normalization. These can be expressed as

$$
\frac{\partial s\left(x, \varepsilon^{\prime}\right)}{\partial x}=-\left(\frac{\partial F_{Z \mid X=x}(z)}{\partial z}\right)^{-1}\left(\frac{\partial F_{Z \mid X=x}(z)}{\partial x}\right)
$$

where $z=s\left(x, \varepsilon^{\prime}\right)$ and as

$$
\begin{aligned}
& s\left(x_{1}, \varepsilon\right)-s\left(x_{0}, \varepsilon\right) \\
= & F_{Z \mid X=x_{1}}^{-1}\left(F_{Z \mid X=x_{0}}\left(z_{0}\right)\right)-z_{0} .
\end{aligned}
$$

See Matzkin (2007) for details. We next consider assumptions under which $U_{z}$ is identified. We start with a nonidentification result that motivates our identification analysis.

\subsection{A Nonidentification Result}

Given $s(x, \varepsilon)$ and $P(z)$, we seek to identify the marginal utility function $U_{z}$. In equilibrium, this function satisfies the first order condition

$$
U_{z}(s(x, \varepsilon), x, \varepsilon)=P_{z}(s(x, \varepsilon)) .
$$

Note that the marginal utility function is identified for those values of $(z, x, \varepsilon)$ that lie on the surface $\{(z, x, \varepsilon): z=s(x, \varepsilon)\}$. On this surface, the value of the marginal utility $U_{z}$ is known, since it must equal the value of the marginal price function.

However, it is clear from this expression that without further restrictions, it is not possible 
to identify the function $U_{z}$ for all values of $(z, x, \varepsilon)$ using data from a single market. For any arbitrary values of $x$ and $\varepsilon$, the value $s(x, \varepsilon)$, the first argument of the function, is uniquely determined. Thus, even if we could observe $\varepsilon$, we could not independently vary $(z, x, \varepsilon)$ and trace out the function on its $n_{x}+2$ dimensional domain. ${ }^{12}$

We offer three responses to this fundamental nonidentification problem: 1) Focus attention on features of $U_{z}$ that are identified; 2) Impose functional restrictions on $U_{z}$ that enable analysts to overcome the exact functional dependence between $z, x$, and $\varepsilon$ that is implied by economic theory; or 3) Obtain data from equilibria in different markets and make use of independent variation in hedonic equilibrium prices across markets. We pursue the first two approaches in the remainder of this section and develop the third approach in Section 4.

\subsection{What is Identified without Further Structure}

Even though $U_{z}$ is not identified using data from a single market, some features of the function $U_{z}$ can be identified. A revealed preference argument shows that the hedonic price function provides a bound on $U_{z}$. If a worker $\left(x_{0}, \varepsilon_{0}\right)$ chooses $z_{0}$ and not $z_{1}$ where $z_{0}>z_{1}$, then it must be the case that

$$
\begin{aligned}
P\left(z_{0}\right)-P\left(z_{1}\right) & \geq U\left(z_{0}, x_{0}, \varepsilon_{0}\right)-U\left(z_{1}, x_{0}, \varepsilon_{0}\right) \\
& =\int_{z_{1}}^{z_{0}} U_{z}\left(s, x_{0}, \varepsilon_{0}\right) d s
\end{aligned}
$$

Differences in hedonic prices for $z_{0}$ and $z_{1}$ in general overestimate the change in welfare between $z_{1}$ and $z_{0} \cdot{ }^{13}$

Other features of $U_{z}$ are point identified. For example, if $x$ contains two variables, $x_{1}$ and $x_{2}$, the ratio of the partial derivatives of $U_{z}$ with respect to $x_{1}$ and $x_{2}$ is identified. To see this, note that one can totally differentiate equation (3.1) with respect to $x_{1}$ and $x_{2}$ to obtain

$$
U_{z z}\left(z, x_{1}, x_{2}, \varepsilon\right) \frac{\partial s\left(x_{1}, x_{2}, \varepsilon\right)}{\partial x_{1}}+U_{z x_{1}}\left(z, x_{1}, x_{2}, \varepsilon\right)=P_{z z}\left(s\left(x_{1}, x_{2}, \varepsilon\right)\right) \frac{\partial s\left(x_{1}, x_{2}, \varepsilon\right)}{\partial x_{1}}
$$

\footnotetext{
${ }^{12}$ This is the nonparametric generalization of the nonidentification result reported in Brown and Rosen (1982).

${ }^{13}$ See Scotchmer (1985), Kanemoto (1988), and Griffith and Nesheim (2008).
} 
and

$$
U_{z z}\left(z, x_{1}, x_{2}, \varepsilon\right) \frac{\partial s\left(x_{1}, x_{2}, \varepsilon\right)}{\partial x_{2}}+U_{z x_{2}}\left(z, x_{1}, x_{2}, \varepsilon\right)=P_{z z}\left(s\left(x_{1}, x_{2}, \varepsilon\right)\right) \frac{\partial s\left(x_{1}, x_{2}, \varepsilon\right)}{\partial x_{2}}
$$

Hence, from the assumed properties of $U$, and the additional assumption that $U_{z z}-P_{z z}$ is non-zero,

$$
\frac{U_{z x_{1}}\left(z, x_{1}, x_{2}, \varepsilon\right)}{U_{z x_{2}}\left(z, x_{1}, x_{2}, \varepsilon\right)}=\left.\frac{\frac{\partial s\left(x_{1}, x_{2}, \varepsilon\right)}{\partial x_{1}}}{\frac{\partial s\left(x_{1}, x_{2}, \varepsilon\right)}{\partial x_{2}}}\right|_{s\left(x_{1}, x_{2}, \varepsilon\right)=z} .
$$

Since $s(x, \varepsilon)$ is identified, the ratios of partial derivatives in (3.2) are identified without any further restrictions. The ratio on the left side of (3.2) measures the effect on $U_{z}$ of changing $x_{1}$ relative to changing $x_{2}$. Since $U_{z}$ is the marginal willingness to accept (WTA) for the worker who chooses $z$, this ratio measures the relative impact on WTA of $x_{1}$ and $x_{2}$. For example, when $z$ is injury risk and $x_{1}$ and $x_{2}$ are education and experience respectively, the ratio measures the relative impacts on WTA of education and experience. This identification result requires no further restrictions on the set of admissible $U_{z}$ functions other than what has been assured thus far. It does not require any normalization of the $s$ function.

Alternatively, if $x$ is scalar and one assumes that the distribution of $\varepsilon$ is known, the same arguments can be used to show that the ratio of partial derivatives

$$
\frac{U_{z x}(z, x, \varepsilon)}{U_{z \varepsilon}(z, x, \varepsilon)}=\left.\frac{\frac{\partial s(x, \varepsilon)}{\partial x}}{\frac{\partial s(x, \varepsilon)}{\partial \varepsilon}}\right|_{s(x, \varepsilon)=z}
$$

is identified without any further restrictions on $U_{z}$. In this case, (3.3) can be used to evaluate the relative impacts on $U_{z}$ of observable $x$ and unobservable $\varepsilon$ for different values of $x$ and at different quantiles of the distribution of $\varepsilon$. In the job injury example, this ratio could be used to study the impact of education on WTA for injury risk at different quantiles of the distribution.

\subsection{Identification Assuming Further Information about the Struc- ture of Preferences and Technology}

A second way to deal with the fundamental nonidentification problem is to assume additional restrictions on the set of admissible $U_{z}$ functions. For example, Ekeland, Heckman, and Nesheim (2004) overcome the nonidentification problem by imposing an additive structure 
on $U_{z}$. In their leading example, they assume that

$$
U_{z}(z, x, \varepsilon)=u_{0}(z)-u_{1}(x)-\varepsilon .
$$

This assumption implies that heterogeneity shifts the marginal utility of $z$ but has no effect on higher order derivatives. All workers have the same curvature in the utility function.

Under this restriction (and similar restrictions discussed in Ekeland, Heckman, and Nesheim (2004)), equation (3.1) reduces to

$$
u_{0}(s(x, \varepsilon))-u_{1}(x)-\varepsilon=P_{z}(s(x, \varepsilon)) .
$$

The additive restriction reduces the dimension of the identification problem by one. Ekeland, Heckman, and Nesheim (2004) show that this implies that $u_{0}, u_{1}$ and the distribution of $\varepsilon$ are identified up to scale and location parameters.

However, the additive structure may be too strong for certain economic environments. From an economic perspective it minimizes the role of heterogeneity in hedonic models. Moreover, it imposes testable restrictions on observable data. Under the additive model restriction, the ratios in (3.2) reduce to

$$
\frac{\frac{\partial u_{1}}{\partial x_{1}}\left(x_{1}, x_{2}\right)}{\frac{\partial u_{1}}{\partial x_{2}}\left(x_{1}, x_{2}\right)}=\left.\frac{\frac{\partial s\left(x_{1}, x_{2}, \varepsilon\right)}{\partial x_{1}}}{\frac{\partial s\left(x_{1}, x_{2}, \varepsilon\right)}{\partial x_{2}}}\right|_{s\left(x_{1}, x_{2}, \varepsilon\right)=z} .
$$

The rate of trade-off between $x_{1}$ and $x_{2}$ must be the same for all quantiles of the $z$ distribution. This is a testable restriction. In the case of scalar $x$, we have

$$
\frac{\partial u_{1}(x)}{\partial x}=\left.\frac{\frac{\partial s(x, \varepsilon)}{\partial x}}{\frac{\partial s(x, \varepsilon)}{\partial \varepsilon}}\right|_{s(x, \varepsilon)=z} .
$$

The impact of $x$ on $z$ must be the same for all $\varepsilon$.

The additive model structure is over-identifying. It is desirable to investigate weaker conditions for identification that are just identifying. Proceeding along these lines, we develop three theorems. Each introduces a different assumption on marginal utility, but all have the effect of reducing the number of arguments of $U_{z}$ by at least one to overcome the nonidentification result of Section 3.1. We emphasize that all are weaker than assumptions imposed in parametric hedonic models or in Ekeland, Heckman, and Nesheim (2004). All impose some separability structure on the utility or production functions and some sort of 
scale normalization. The types of restrictions required are similar in spirit to either those required to identify household equivalence scales in classical demand theory or to restrictions used in random coefficients models. See Lewbel (1989) or Lewbel (1997) for related results on identification of household equivalence scales and a survey of that literature.

\subsubsection{Observable Scales}

For our first theorem, we assume that $U_{z}$ is a weakly separable function of the pair $(z, x)$, i.e., for some functions $m: R^{2} \rightarrow R$, and $q: R^{2} \rightarrow R, \quad U_{z}(z, x, \varepsilon)=m(q(z, x), \varepsilon)$. Moreover, we assume that the function, $q$, which aggregates the effect of $z$ and $x$ on $U_{z}$ is known. In the job risk example, $q(z, x)$ can be interpreted as the observable scale or quality of the job-worker match while $\varepsilon$ is unobserved heterogeneity in marginal utility. Note that when $q(z, x)=z / x$, our specification is consistent with Barten's (1964) scale model for utility. Since $m$ is nonadditive in $\varepsilon$, a normalization on either $m$ or the distribution of $\varepsilon$ is also needed. In the next theorem, we assume that the distribution of $\varepsilon$ is specified a priori. ${ }^{14}$

Theorem 3.1 Suppose that for some unknown differentiable function $m: \mathbf{R}^{2} \rightarrow \mathbf{R}$, which is strictly decreasing in its second argument, and some known differentiable function $q: \mathbf{R}^{2} \rightarrow$ $\mathbf{R}$, the marginal utility function can be written

$$
U_{z}(z, x, \varepsilon)=m(q(z, x), \varepsilon)
$$

Further, assume that $F_{\varepsilon}$ is known and let $\left(q^{l}(\varepsilon), q^{u}(\varepsilon)\right)$ denote the support of $q(s(x, \varepsilon), x)$ for any $\varepsilon \in \widetilde{E}$. Then, for all $\varepsilon$ and all $x$ such that $q(s(x, \varepsilon), x) \in\left(q^{l}(\varepsilon), q^{u}(\varepsilon)\right), U_{z}(z, x, \varepsilon)$ is identified.

\section{Proof. See Appendix A.}

The intuition for this result is that for a given value of $\varepsilon$, identification of $s(x, \varepsilon)$ allows one to find all pairs of $(z, x)$ that are consistent with that value of $\varepsilon$. The separability restriction on $U_{z}$ allows one to select from among these pairs the one that produces a given value of $q(z, x)$. Combining these two results allows one to identify $U_{z}$ at any arbitrary point.

Theorem 3.1 uses a separability restriction and a normalization of the distribution of $\varepsilon$. An alternative approach is to invoke separability, but to normalize the function $U_{z}$, instead

\footnotetext{
${ }^{14}$ For example, since $\varepsilon$ is not observed, a natural choice is to specify that $\varepsilon$ is uniformly distributed. Thus $\varepsilon$ can be interpreted as the quantile in the unobserved heterogeneity distribution.
} 
of the distribution of $\varepsilon$, by assuming that its value is known at a point. Along these lines, we obtain the following theorem.

Theorem 3.2 Let $x \in \mathbf{R}$. Suppose that for some unknown, differentiable function $m: \mathbf{R}^{2} \rightarrow$ $\mathbf{R}$, which is strictly decreasing in its last argument, and some known, differentiable, function $q: \mathbf{R}^{2} \rightarrow \mathbf{R}$,

$$
U_{z}(z, x, \varepsilon)=m(q(z, x), \varepsilon) .
$$

Use the function $P_{z}$ to fix the value of the unknown function $U_{z}$ at one value $\bar{x}$ of $x$, and on the 45 degree line on the $(z, \varepsilon)$ space, by requiring that for all $t$,

$$
U_{z}(t, \bar{x}, t)=P_{z}(t)
$$

Let $\varepsilon$ be given. Let $q \in\left(q^{l}(\varepsilon), q^{u}(\varepsilon)\right)$, the support of $q(s(x, \varepsilon), x)$. Then, for $x$ such that $q(s(x, \varepsilon), x) \in\left(q^{l}(\varepsilon), q^{u}(\varepsilon)\right), U_{z}(z, x, \varepsilon)$ is identified.

Proof. See Appendix A.

\subsubsection{Random Coefficients}

Results analogous to Theorems 3.1 and 3.2 can be obtained if, instead of requiring that $U_{z}$ be separable in $(z, x)$, one assumes that $U_{z}$ is separable in $(z, \varepsilon)$. In other words, one can assume that for some unknown function $m: \mathbf{R}^{2} \rightarrow \mathbf{R}$, which is strictly increasing in its first argument, and some known function $q: \mathbf{R}^{2} \rightarrow \mathbf{R}$, which is strictly decreasing in its second argument

$$
U_{z}(z, x, \varepsilon)=m(q(z, \varepsilon), x) .
$$

This specification is a generalization of a linear random coefficients model. When $q(z, \varepsilon)=$ $\varepsilon \cdot z$, this specification is consistent with a linear random coefficients model for the marginal utility. $^{15}$

A result analogous to that of Theorem 3.1 can be obtained when $F_{\varepsilon}$ is known. For any $x$, let $\left(q_{l}(x), q_{u}(x)\right)$ denote the support of $q(s(x, \varepsilon), \varepsilon)$. Following an argument similar to that used in the proof of Theorem 3.1, it is easy to show that for all $x$ and all $\varepsilon$ such that $q(s(x, \varepsilon), \varepsilon) \in\left(q^{l}(x), q^{u}(x)\right), U_{z}(z, x, \varepsilon)$ is identified. The result that corresponds to Theorem

\footnotetext{
${ }^{15}$ The scalar version of the parametric demand model analyzed in Bajari and Benkard (2005) is a special case of this model. They assume that $m$ is parametric.
} 
3.2 is obtained by fixing the values of $m$ when $x=\bar{x}$, a specified value of $x$, as

$$
m(q(t, t), \bar{x})=P_{z}(t)
$$

\subsubsection{Multiple Observed Scales}

When $x$ is a vector, alternative restrictions can be used to achieve identification. As an example, when $x$ is a two-dimensional vector $\left(x_{1}, x_{2}\right)$, we can impose the restriction that $U_{z}$ is weakly separable into two known functions, $q_{1}\left(z, x_{1}\right)$ and $q_{2}\left(x_{2}, \varepsilon\right)$. The next theorem establishes that this restriction, along with a normalization on $U_{z}$, which is weaker than what is required in the case where $x$ is a scalar, allows one to identify $U_{z}$.

Theorem 3.3 Let $x=\left(x_{1}, x_{2}\right) \in \mathbf{R}^{2}$. Suppose that for some unknown differentiable function $m: \mathbf{R}^{2} \rightarrow \mathbf{R}$, which is strictly increasing in its second argument, and some known differentiable functions $q_{1}: \mathbf{R}^{2} \rightarrow \mathbf{R}$ and $q_{2}: \mathbf{R}^{2} \rightarrow \mathbf{R}$

$$
U_{z}\left(z, x_{1}, x_{2}, \varepsilon\right)=m\left(q_{1}\left(z, x_{1}\right), q_{2}\left(x_{2}, \varepsilon\right)\right)
$$

where $q_{2}$ is strictly decreasing in its second argument. Let $\left[q_{2}^{l}, q_{2}^{u}\right]$ denote the support of $q_{2}\left(x_{2}, \varepsilon\right)$. Assume that the function $m$ is known at one point $\left(\bar{z}, \bar{x}_{1}, \bar{\alpha}\right)$ where $\bar{\alpha} \in\left[q_{2}^{l}, q_{2}^{u}\right]$, so that

$$
m\left(q_{1}\left(\bar{z}, \bar{x}_{1}\right), \bar{\alpha}\right)=P_{z}(\bar{z}) .
$$

For any $t_{2} \in\left[q_{2}^{l}, q_{2}^{u}\right]$, let $\left[q_{1}^{l}\left(t_{2}\right), q_{1}^{u}\left(t_{2}\right)\right]$ denote the support of $q_{1}\left(s\left(x_{1}, x_{2}, \varepsilon\right), x_{1}\right)$ conditional on $q_{2}\left(x_{2}, \varepsilon\right)=t_{2}$. Then, for any $\left(z, x_{1}, x_{2}, \varepsilon\right)$ such that $q_{2}\left(x_{2}, \varepsilon\right) \in\left[q_{2}^{l}, q_{2}^{u}\right]$ and $q_{1}\left(z, x_{1}\right) \in$ $\left[q_{1}^{l}\left(t_{2}\right), q_{1}^{u}\left(t_{2}\right)\right], U_{z}\left(z, x_{1}, x_{2}, \varepsilon\right)$ is identified.

Proof. See Appendix A.

Identification of $U_{z}$ is obtained from a sequence of arguments. First, equation (3.7) implies that the supply function $s\left(x_{1}, x_{2}, \varepsilon\right)$ is a weakly separable function of $x_{1}$ and $q_{2}\left(x_{2}, \varepsilon\right)$. Equation (3.8) implies that the supply function is known at one point. Further, the strict monotonicity of $m$ and $q_{2}$ in their second arguments implies that the supply function is strictly increasing in $\varepsilon$. These implications guarantee that the supply function $s$ and the distribution of $\varepsilon$ are identified. Next, to identify the value of $m\left(t_{1}, t_{2}\right)$ at an arbitrary point $\left(t_{1}, t_{2}\right)$ on the relevant domain, we first find values $x_{1}^{*}, x_{2}^{*}$, and $\varepsilon^{*}$ such that when $z=s\left(x_{1}^{*}, x_{2}^{*}, \varepsilon^{*}\right), q_{1}\left(z, x_{1}^{*}\right)=t_{1}$ and $q_{2}\left(x_{2}^{*}, \varepsilon^{*}\right)=t_{2}$. Finally, since such a $z$ satisfies the FOC, it follows that $m\left(t_{1}, t_{2}\right)=P_{z}(z)=P_{z}\left(s\left(x_{1}^{*}, x_{2}^{*}, \varepsilon^{*}\right)\right)$. Thus, independent variation in $x_{1}$ and 
$x_{2}$, the assumed dependence of $U_{z}$ on only two arguments, and knowledge of the functions $q_{1}$ and $q_{2}$, allow one to trace out $U_{z}$ as a function of its two arguments.

The statement and proof of Theorem 3.3 can easily be modified to show that the function $U_{z}$ is also identified when it can be expressed as a function $m\left(t_{1}, x_{1}\right)$, where $t_{1}=q_{1}\left(z, t_{2}\right)$ and $t_{2}=q_{2}\left(x_{2}, \varepsilon\right)$. To see this, suppose that for some unknown function $m: \mathbf{R}^{2} \rightarrow \mathbf{R}$ and some known functions $q_{1}: \mathbf{R}^{2} \rightarrow \mathbf{R}$ and $q_{2}: \mathbf{R}^{2} \rightarrow \mathbf{R}$, such that $m$ is strictly increasing in its first argument, $q_{1}$ is strictly increasing in its second argument, and $q_{2}$ is strictly decreasing in its second argument

$$
U_{z}\left(z, x_{1}, x_{2}, \varepsilon\right)=m\left(q_{1}\left(z, q_{2}\left(x_{2}, \varepsilon\right)\right), x_{1}\right)
$$

Assume that the function $m$ is known at one point $\left(\bar{z}, \bar{x}_{1}, \bar{\alpha}\right)$ so that

$$
m\left(q_{1}(\bar{z}, \bar{\alpha}), \bar{x}_{1}\right)=P_{z}(\bar{z})
$$

Then, as in the proof of Theorem 3.3, it can be shown by using (3.9) that the supply function, $s\left(x_{1}, x_{2}, \varepsilon\right)$ is weakly separable in $q_{2}\left(x_{2}, \varepsilon\right)$. By (3.10), the value of $s$ is fixed at one point. By the monotonicity of $m$ and $q_{1}, s$ is strictly increasing in $q_{2}$. These properties guarantee identification of $s$ and of the distribution of $\varepsilon$ using the analysis of Matzkin (1999) and Matzkin (2003). To identify the value of $m\left(t_{1}, t_{2}\right)$ at an arbitrary vector $\left(t_{1}, t_{2}\right)$, let $x_{1}^{*}=t_{2}$, and find $x_{2}^{*}$, and $\varepsilon^{*}$ such that when $z=s\left(x_{1}^{*}, x_{2}^{*}, \varepsilon^{*}\right), q_{1}\left(z, q_{2}\left(x_{2}^{*}, \varepsilon^{*}\right)\right)=t_{1}$. Then, as in the previous argument, $m\left(t_{1}, t_{2}\right)=P_{z}(z)=P_{z}\left(s\left(x_{1}^{*}, x_{2}^{*}, \varepsilon^{*}\right)\right)$.

\subsubsection{Economic Implications of the Restrictions}

Consider the economic implications of some of the restrictions that we have invoked. We focus on the restrictions used in Theorems 3.1 and 3.2. Consider the alternative specifications

$$
U_{z}(z, x, \varepsilon)=m_{1}(z x, \varepsilon)
$$

and

$$
U_{z}(z, x, \varepsilon)=m_{2}(z \varepsilon, x)
$$

These models make different assumptions about how heterogeneity impacts marginal utility. Model (3.11) imposes the requirement that $\frac{U_{z z}}{U_{z x}}=\frac{x}{z}$ while model (3.12) imposes the requirement that $\frac{U_{z z}}{U_{z \varepsilon}}=\frac{\varepsilon}{z}$. The former condition might be appropriate when the researcher has prior information about the marginal rate of substitution between $z$ and observable $x$ 
and but has no information about how unobserved heterogeneity affects marginal utility. The latter might be appropriate if the researcher is willing to impose a random coefficients structure but has no prior information about the effects of observables.

\section{Identification in Multiple Markets}

The possibilities for identification of $U_{z}(z, x, \varepsilon)$ in a single market are limited because all workers face the same price schedule. Across multiple markets, the marginal price function $P_{z}(z)$ will typically vary depending on underlying market conditions. For example, assuming that the marginal utility function, $U_{z}(z, x, \varepsilon)$, does not vary across markets, the marginal price function (and the supply function $s(x, \varepsilon)$ ) will, in general, vary across markets when the distributions of worker or firm attributes vary across markets. When data are available from multiple markets and cross-market variation in the distributions of observables causes crossmarket variation in $P_{z}(z)$ and $s(x, \varepsilon)$, such variation can be used to identify the function $U_{z}(z, x, \varepsilon)$ without invoking the conditions of Section 3 .

Our analysis provides a general approach to identification of $U_{z}$ under weaker conditions than are required when using data from a single cross-section. Use of multimarket data to identify hedonic models is proposed in Brown and Rosen (1982), Brown (1983) and Epple (1987). Our analysis is more general than theirs because we consider the nonseparable case whereas their analyses assume linearity of supply and price equations. Also, our approach makes explicit that the equilibrium price and the supply function depend on the distributions of observable characteristics of firms and workers.

Suppose that the distributions of $\varepsilon$ and $\eta$ are the same in all markets. Further assume that the distributions of $x$ and $y$ with densities denoted by $\left(f_{x}, f_{y}\right) \in \mathcal{F}(\widetilde{X}) \times \mathcal{F}(\widetilde{Y}) \subseteq$ $L_{2}\left(\tilde{X}, \mu_{x}\right) \times L_{2}\left(\widetilde{Y}, \mu_{y}\right)$ vary across markets. Here, $\mu_{x}$ and $\mu_{y}$ are Lebesgue measure on $\widetilde{X}$ and $\widetilde{Y}$ respectively and $L_{2}$ represents the space of square integrable functions. Suppose that a multimarket sample exists from $M$ markets with $N_{m}$ observations on $(z, y, x)$ from each market $m$. The marginal price and supply functions in each market will depend on $\left(f_{x}^{m}, f_{y}^{m}\right)$, the densities of observed $x$ and $y$ in each market. Dropping superscripts, write these functions as $P_{z}\left(z, f_{x}, f_{y}\right)$ and $s\left(x, \varepsilon, f_{x}, f_{y}\right)$.

The functions $\left(f_{x}, f_{y}\right)$ and the functional $P_{z}\left(z, f_{x}, f_{y}\right)$ are identified in a multimarket sample satisfying these conditions. Using results in Matzkin (1999, 2003), the functional $s\left(x, \varepsilon, f_{x}, f_{y}\right)$ is nonparametrically identified. Hence, multimarket data allow one to identify 
the distributions of the observables and the dependence of the marginal price and the supply function on these distributions. We use this information to identify the marginal utility function $U_{z}(z, x, \varepsilon)$ in the following way.

From the workers' first order condition:

$$
U_{z}\left(s\left(x, \varepsilon, f_{x}, f_{y}\right), x, \varepsilon\right)=P_{z}\left(s\left(x, \varepsilon, f_{x}, f_{y}\right), f_{x}, f_{y}\right) .
$$

We have made explicit the dependence of $P_{z}$ and $s$ on $f_{x}$ and $f_{y}$. In a single cross section, the price function and the supply function are fixed and we cannot independently vary the three arguments of $U_{z}$. With multimarket data, both $P_{z}$ and $s$ vary for each $(x, \varepsilon)$ provided that $f_{x}, f_{y}$, or both, vary across markets.

Before stating and proving Theorem 4.1, we need to introduce some new notation. Given $\left(f_{x}^{m}, f_{y}^{m}\right) \subseteq \mathcal{F}(\tilde{X}) \times \mathcal{F}(\tilde{Y})$, let $s^{m}(x, \varepsilon)$ and $P^{m}(z)$ be, respectively, the supply function and price function implied by equilibrium condition (2.5). Further, let

$$
S=\left\{(z, x, \varepsilon): z=s^{m}(x, \varepsilon) \text { for some }\left(f_{x}^{m}, f_{y}^{m}\right) \subseteq \mathcal{F}(\widetilde{X}) \times \mathcal{F}(\widetilde{Y})\right\} .
$$

Similarly, let $d^{m}(y, \eta)$ be the equilibrium demand function and

$$
D=\left\{(z, y, \eta): z=d^{m}(y, \eta) \text { for some }\left(f_{x}^{m}, f_{y}^{m}\right) \subseteq \mathcal{F}(\widetilde{X}) \times \mathcal{F}(\widetilde{Y})\right\} .
$$

The sets $S$ and $D$ are the sets of matches that could occur in a feasible equilibria.

Theorem 4.1 Let $S$ and $D$ be defined as in (4.1) and (4.2). Assume that $\left(f_{\varepsilon}, f_{\eta}\right)$ are known and are the same across markets, then $U_{z}(z, x, \varepsilon)$ is identified for all $(z, x, \varepsilon) \in S$ and $\Gamma_{z}(z, y, \eta)$ is identified for all $(z, y, \eta) \in D$.

Proof. Let $(z, x, \varepsilon) \in S$. Then $z=s^{m}(x, \varepsilon)$ for some market $m$ with $\left(f_{x}^{m}, f_{y}^{m}\right) \subseteq \mathcal{F}(\tilde{X}) \times$ $\mathcal{F}(\tilde{Y})$ and $U_{z}(z, x, \varepsilon)=P_{z}^{m}\left(s^{m}(x, \varepsilon)\right)$ where $P_{z}^{m}(z)$ is the marginal price in market $m$. Thus, $U_{z}$ is identified at all points $(z, x, \varepsilon)$ such that $z$ is an equilibrium choice for $(x, \varepsilon)$ in some feasible equilibrium. By an identical argument, $\Gamma_{z}(z, y, \eta)$ is identified for all $(z, y, \eta) \in$ $D$.

Theorem 4.1 exploits the variation in price and supply functions induced by cross-market variation in the distributions of observables. Given a fixed pair $\left(f_{x}^{0}, f_{y}^{0}\right)$, under the conditions stated in section 2, equation (2.5) will have a unique solution $P^{0}(z)$. This solution will imply a pair of supply and demand functions $s^{0}(x, \varepsilon)$ and $d^{0}(y, \eta)$. Perturbing the underlying 
distributions to $\left(f_{x}^{1}, f_{y}^{1}\right)$ will result in a new equilibrium price function $P^{1}(z)$ and new supply and demand functions $s^{1}(x, \varepsilon)$ and $d^{1}(y, \eta)$. In general, if the dependence of utility and production functions on $y$ and $x$ is not degenerate, it will be true that $P^{1}(z) \neq P^{0}(z)$, $s^{1}(x, \varepsilon) \neq s^{0}(x, \varepsilon)$ and $d^{1}(y, \eta) \neq d^{0}(y, \eta)$.

\section{Estimation}

Since our identification results are constructive, they suggest estimators for the marginal utility (resp. production) functions and their distributions, based on estimators for the derivative of the price function and the supply (resp. demand) function. The derivative of the price function can be estimated as the derivative of the kernel regression of price on $z$. The demand and supply functions, and the distributions of the unobservable variables, can be estimated also using kernel methods, as described in Matzkin (2003).

The innovation in this paper over previous research is the estimation of the marginal utility and marginal product functions. Following our proofs of identification, we can construct estimators for either the supply or demand side. Consider, for example, a variant of the specification of the marginal utility in Theorem 3.3. Suppose that for some unknown function $m$ and known functions $q_{1}$ and $q_{2}$,

$$
U_{z}\left(z, x_{1}, x_{2}, x_{3}, \varepsilon\right)=m\left(q_{1}\left(z, x_{1}\right), q_{2}\left(x_{2}, \varepsilon\right), x_{3}\right)
$$

The vector, $x_{3}$, of additional observable individual characteristics, is included here to allow for the possibility that the dimension of $x$ is larger than 2. Note, however, that $x_{3}$ is not needed for our main identification results. The supply function resulting from this specification is separable in $q_{2}\left(x_{2}, \varepsilon\right)$. An estimator for such supply function, denoted by $\widehat{s}\left(x_{1}, q_{2}\left(x_{2}, \varepsilon\right), x_{3}\right)$, can be derived following Matzkin (2003). Let $\widehat{P}_{z}(z)$ denote the kernel estimator for the derivative of the price function, and let $t_{1}, t_{2}$ be arbitrary values. It follows from the proof of Theorem 3.3 that

$$
\widehat{m}\left(t_{1}, t_{2}, x_{3}\right)=\widehat{P}_{z}\left(\widehat{s}\left(\widehat{x}_{1}^{*}, t_{2}, x_{3}\right)\right)
$$

where $\widehat{x}_{1}^{*}$ is the value of $x_{1}$ that solves the equation

$$
q_{1}\left(\widehat{s}\left(x_{1}, t_{2}, x_{3}\right), x_{1}\right)=t_{1} .
$$


Equation (5.1) with the estimated $\widehat{P}_{z}$ and $\widehat{s}$ plugged in is our estimator of the marginal utility function in this model.

Let $x_{1}^{*}$ denote the value of $x_{1}$ that satisfies

$$
q_{1}\left(s\left(x_{1}^{*}, t_{2}, x_{3}\right), x_{1}^{*}\right)=t_{1} .
$$

We demonstrate in Web Appendix D that, under standard conditions, the rate of convergence of $\widehat{x}_{1}^{*}$ to $x_{1}^{*}$ is the same as that of $\widehat{s}$ to $s$. We develop the results using the Implicit Function Theorem in Banach spaces of Hildebrandt and Graves (1927), to derive a first order Taylor type approximation for the functional that maps the estimated distribution of the observable variables into the value of $\widehat{x}_{1}^{*}$. Note that because $q_{1}$ is known and $\left(t_{1}, t_{2}\right)$ is given, equation (5.2) implies that $\widehat{s}\left(\widehat{x}_{1}^{*}, t_{2}, x_{3}\right)$ is a deterministic function of $\widehat{x}_{1}^{*}$. Hence, the asymptotic behavior of $\widehat{s}\left(\widehat{x}_{1}^{*}, t_{2}, x_{3}\right)$ can be derived from that of $\widehat{x}_{1}^{*}$ using well known techniques. Also note that, when the dimension of the vector $\left(x_{1}, x_{2}, x_{3}\right)$ is larger than the dimension of $z$ plus 2 , we may assume for the purpose of analyzing the asymptotic behavior of $\widehat{P}_{z}\left(\widehat{s}\left(\widehat{x}_{1}^{*}, t_{2}, x_{3}\right)\right)$ that $P_{z}$ is known. Hence, under standard regularity conditions, $\widehat{m}\left(t_{1}, t_{2}, x_{3}\right)$ will converge to $m\left(t_{1}, t_{2}, x_{3}\right)$ at the same rate that $\widehat{s}$ converges to $s$, with an asymptotic variance that can be calculated by that of $\widehat{x}_{1}^{*}$ using the standard Delta method. See Web Appendix D for additional detail and Web Appendix E for some simulations that suggest that the method is practical and works well in practice.

\section{Conclusions}

This paper develops methods for identifying the deep structural parameters of hedonic equilibrium models where both the marginal utility of workers and the marginal product of firms are nonadditive functions of attributes and a random vector of individual characteristics, which are different for the workers and firms. We develop sufficient conditions to identify the marginal utility and marginal product functions using both single market and multimarket data. In the single market case, we develop nonparametric estimators for these functions and show in Web Appendix D that they are consistent and asymptotically normal. Limited Monte Carlo evidence presented in Web Appendix E suggests that the methods work well in practice.

Our analysis is for a hedonic model with a single attribute. However, as discussed in Sections 2 and 3 , these results apply equally to a model in which the hedonic attribute is a 
single index with some known structure in terms of a vector of characteristics. We also note that our methods allow one to deal with unobserved product attributes, as long as these can be identified from the price function, as in Bajari and Benkard (2005), since in such a case one can treat them as observed. In contrast to their analysis, our analysis allows for a nonparametric utility function. More generally, the first order conditions in unrestricted multiple attribute hedonic models are systems of simultaneous equations. General conditions for nonparametric identification of systems of equations are given in Matzkin (2008). Further

work is required to investigate which types of restrictions developed in Matzkin (2008) are most appropriate for hedonic applications.

\section{A Proofs}

Proof of Theorem 3.1. Since model (3.4) satisfies the conditions in section $2, s(x, \varepsilon)$ satisfies (2.1) and (2.2). Thus, $s$ is a nonadditive function in $\varepsilon$ which is strictly increasing in $\varepsilon$. Since $\varepsilon$ is independent of $X$, it follows by Matzkin (1999) and Matzkin (2003) that

$$
s(x, \varepsilon)=F_{Z \mid X}^{-1}\left(F_{\varepsilon}(\varepsilon)\right)
$$

Since $F_{\varepsilon}$ is given, $s$ is identified. Let $\left(t_{1}, t_{2}\right)$ be such that $t_{1} \in\left(q^{l}\left(t_{2}\right), q^{u}\left(t_{2}\right)\right)$. Find $x^{*}$ such that

$$
q\left(s\left(x^{*}, t_{2}\right), x^{*}\right)=t_{1}
$$

Then,

$$
m\left(t_{1}, t_{2}\right)=P_{z}\left(s\left(x^{*}, t_{2}\right)\right)
$$

Proof of Theorem 3.2. As in Theorem 3.1, $s$ is a nonadditive function in $\varepsilon$ which is strictly increasing in $\varepsilon$. Further, it follows from equation (3.5) that the supply function, $s(x, \varepsilon)$, satisfies

$$
s(\bar{x}, \varepsilon)=\varepsilon
$$

Then, by Matzkin (1999)

$$
F_{\varepsilon}(\varepsilon)=F_{Z \mid X=\bar{x}}(\varepsilon)
$$

and

$$
s(x, \varepsilon)=F_{Z \mid X=x}^{-1}\left(F_{Z \mid X=\bar{x}}(\varepsilon)\right) .
$$


To establish that the function $m$ is identified, let $x^{*}$ denote the solution to

$$
q\left(s\left(x^{*}, t_{2}\right), x^{*}\right)=t_{1}
$$

Hence,

$$
\begin{aligned}
m\left(t_{1}, t_{2}\right) & =m\left(q\left(s\left(x^{*}, t_{2}\right), x^{*}\right), t_{2}\right) \\
& =P_{z}\left(s\left(x^{*}, t_{2}\right)\right)
\end{aligned}
$$

Proof of Theorem 3.3. Since $U_{z}$ is weakly separable in $q_{2}\left(x_{2}, \varepsilon\right)$, the function $z=$ $s\left(x_{1}, x_{2}, \varepsilon\right)$ is also weakly separable in $q_{2}\left(x_{2}, \varepsilon\right)$. Hence, for some unknown function $v$

$$
s\left(x_{1}, x_{2}, \varepsilon\right)=v\left(x_{1}, q_{2}\left(x_{2}, \varepsilon\right)\right) .
$$

Further, since all of the conditions in section 2 are satisfied and since $q_{2}$ is strictly increasing in both $x_{2}$ and $\varepsilon, v$ is strictly increasing in its second argument.

Let $x_{2}$ and $\varepsilon$ be such that $q_{2}\left(x_{2}, \varepsilon\right)=\bar{\alpha}$. Then, by separability and condition (3.8) in the statement of the theorem

$$
U_{z}\left(\bar{z}, \bar{x}_{1}, q_{2}\left(x_{2}, \varepsilon\right)\right)=P_{z}(\bar{z})
$$

where $\bar{z}$ satisfies the FOC $(2.1)$ when $x_{1}=\bar{x}_{1}$ and $q_{2}\left(x_{2}, \varepsilon\right)=\bar{\alpha}$. It follows that

$$
s\left(\bar{x}_{1}, x_{2}, \varepsilon\right)=v\left(\bar{x}_{1}, q_{2}\left(x_{2}, \varepsilon\right)\right)=\bar{z} .
$$

It then follows from the analysis of Matzkin (2003) that the function $v$ and the distribution of $\varepsilon$ are identified from the conditional distribution of $Z$ given $X=\left(X_{1}, X_{2}\right)$.

To show that the function $m$ is identified, let $\left(t_{1}, t_{2}\right)$ be any vector such that $t_{2} \in\left[q_{2}^{l}, q_{2}^{u}\right]$ and $t_{1} \in\left[q_{1}^{l}\left(t_{2}\right), q_{1}^{u}\left(t_{2}\right)\right]$. Let $x_{1}^{*}$ denote a solution to

$$
q_{1}\left(v\left(x_{1}^{*}, t_{2}\right), x_{1}^{*}\right)=t_{1}
$$

Since $q_{1}$ is a known function and $v$ can be recovered from the conditional cdf of $z$ given $\left(x_{1}, x_{2}\right)$, the only unknown in the expression is $x_{1}^{*}$. Since $t_{2} \in\left[q_{2}^{l}, q_{2}^{u}\right]$ and $t_{1} \in\left[q_{1}^{l}\left(t_{2}\right), q_{1}^{u}\left(t_{2}\right)\right]$, 
$x_{1}^{*}$ exists. Since $v\left(x_{1}^{*}, t_{2}\right)$ satisfies the FOC $(2.1)$,

$$
\begin{aligned}
m\left(t_{1}, t_{2}\right) & =m\left(q_{1}\left(v\left(x_{1}^{*}, t_{2}\right), x_{1}^{*}\right), t_{2}\right) \\
& =P_{z}\left(v\left(x_{1}^{*}, t_{2}\right)\right) \\
& =P_{z}\left(s\left(x_{1}^{*}, x_{2}^{*}, \varepsilon^{*}\right)\right)
\end{aligned}
$$

for any $x_{2}^{*}$ and $\varepsilon^{*}$ such that $q_{2}\left(x_{2}^{*}, \varepsilon^{*}\right)=t_{2}$. In (A.1), the first equality follows because $q\left(v\left(x_{1}^{*}, t_{2}\right), x_{1}^{*}\right)=t_{1}$. The second equality follows because the value of the marginal utility function $m$ equals the value of the marginal price function at the particular value of $z$ that satisfies the first order conditions. The third equality follows by the restriction on the function $s$. Since the function $P_{z}$ is known and the function $s$ can be recovered without knowledge of $m,(A .1)$ implies that $m$ is identified. 


\section{References}

Bajari, P. and C. Benkard (2005). Demand Estimation with Heterogeneous Consumers and Unobserved Product Characteristics: A Hedonic Approach. Journal of Political Economy 113(6), 1239-1276.

Barten, A. (1964). Family Composition, Prices and Expenditure Patterns. Econometric Analysis for National Economic Planning, 277-292.

Berry, S. and A. Pakes (2007). The pure characteristics demand model*. International Economic Review 48(4), 1193-1225.

Brown, J. N. (1983). Structural estimation in implicit markets. In J. E. Triplett (Ed.), The Measurement of Labor Cost. Chicago: University of Chicago Press for NBER.

Brown, J. N. and H. S. Rosen (1982, May). On the estimation of structural hedonic price models. Econometrica 50(3), 765-768.

Chiappori, P.-A., R. McCann, and L. Nesheim (2008). Hedonic price equilibria, stable matching, and optimal transport: Equivalence, topology, and uniqueness. Forthcoming, Economic Theory.

Ekeland, I. (2005, April). Existence, uniqueness and efficiency of equilibrium in hedonic markets with multidimensional types. University of British Columbia.

Ekeland, I., J. J. Heckman, and L. Nesheim (2004, February). Identification and estimation of hedonic models. Journal of Political Economy 112(S1), S60-S109. Paper in Honor of Sherwin Rosen: A Supplement to Volume 112.

Epple, D. (1987, February). Hedonic prices and implicit markets: Estimating demand and supply functions for differentiated products. Journal of Political Economy 95(1), 59-80.

Epple, D. and H. Sieg (1999). Estimating Equilibrium Models of Local Jurisdictions. Journal of Political Economy 107(4), 645-681.

Gale, D. and H. Nikaido (1965). The Jacobian matrix and global univalence of mappings. Mathematische Annalen 159(2), 81-93.

Griffith, R. and L. Nesheim (2008). Household willingness to pay for organic products. CEMMAP Working Paper CWP18/08. 
Heckman, J. J. and E. J. Vytlacil (2007). Econometric evaluation of social programs, part I: Causal models, structural models and econometric policy evaluation. In J. Heckman and E. Leamer (Eds.), Handbook of Econometrics, Volume 6B, pp. 4779-4874. Amsterdam: Elsevier.

Hildebrandt, T. and L. Graves (1927). Implicit functions and their differentials in general analysis. Transactions of the American Mathematical Society 29(1), 127-153.

Hurwicz, L. (1962). On the structural form of interdependent systems. In E. Nagel, P. Suppes, and A. Tarski (Eds.), Logic, Methodology and Philosophy of Science, pp. 232-239. Stanford University Press.

Kahn, S. and K. Lang (1988, February). Efficient estimation of structural hedonic systems. International Economic Review 29(1), 157-166.

Kanemoto, Y. (1988, July). Hedonic prices and the benefits of public projects. Econometrica 56(4), 981-989.

Kniesner, T. J. and J. D. Leeth (1995). Simulating Workplace Safety Policy. Boston, MA: Kluwer Academic Publishers.

Leeth, J. D. and J. Ruser (2003). Compensating wage differentials for fatal and nonfatal risk by gender and race. Journal of Risk and Uncertainty 27(3), 257-277.

Lewbel, A. (1989). Identification and Estimation of Equivalence Scales under Weak Separability. Review of Economic Studies 56(2), 311-16.

Lewbel, A. (1997). Consumer Demand Systems and Household Equivalence Scales. Handbook of Applied Econometrics 2, 167-201.

Matzkin, R. L. (1999). Nonparametric estimation of nonadditive random functions. First edition. Unpublished manuscript, Northwestern University, Department of Economics.

Matzkin, R. L. (2003, September). Nonparametric estimation of nonadditive random functions. Econometrica 71(5), 1339-1375.

Matzkin, R. L. (2007). Nonparametric identification. In J. Heckman and E. Leamer (Eds.), Handbook of Econometrics, Volume 6B. Amsterdam: Elsevier. 
Matzkin, R. L. (2008). Identification in nonparametric simultaneous equations models. Econometrica 76(5), 945-978.

Rosen, S. (1974, January-February). Hedonic prices and implicit markets: Product differentiation in pure competition. Journal of Political Economy 82(1), 34-55.

Scotchmer, S. (1985, October). Hedonic prices and cost-benefit analysis. Journal of Economic Theory 37(1), 55-75.

Sieg, H., V. K. Smith, H. S. Banzhaf, and R. Walsh (2004, November). Estimating the general equilibrium benefits of large changes in spatially delineated public goods. International Economic Review 45(4), 1047-1077. 